\title{
A Temporal Area Variation Regularized Deep Learning Network for Left Ventricle Segmentation on CMR
}

\author{
Gongning Luo ${ }^{1}$, Kuanquan Wang ${ }^{1 *}$, Shaodong $\mathrm{Cao}^{2}$, Qince $\mathrm{Li}^{1}$, Henggui Zhang ${ }^{1,3}$ \\ ${ }^{1}$ Harbin Institute of Technology, Harbin, China \\ ${ }^{2}$ The Fourth Hospital of Harbin Medical University, Harbin, China \\ ${ }^{3}$ University of Manchester, Manchester, UK
}

\begin{abstract}
The automated segmentation of the left ventricular $(L V)$ on MRI is a crucial step for the evaluation of LV structure and function. However, $L V$ segmentation is still a challenging task, mainly because of inherent difficulties from the variable imaging conditions. Hence this study aims to propose an innovative approach to segment $L V$ endocardium and epicardium based on the phenomena of temporal area variation correlation. The proposed method is three-fold: (1) For the first time, we formulated a significant phenomenon that epicardium and endocardium have same area variation tendency into a temporal area variation constraint. (2) We designed a deep leaning network based on RNN to model such a temporal area variation constraint. (3) An efficient optimization framework was developed to achieve end-toend optimization. The deep learning network was trained and validated on cardiac MRI datasets from MICCAI 2012 LV segmentation challenge including 100 patients (50 train patients and 50 test patients).
\end{abstract}

\section{Introduction}

The cardiac disease gets more and more attention because of that it is the leading killer of human beings. Hence, the cardiac diseases get the more attention of researchers in recent years [1-3]. The left ventricle (LV) chamber is the biggest cardiac tissue, so most of research focuses on automated LV segmentation and quantification research [4]. The function indexes, i.e., LV mass, LV volume, and eject fraction (EF) are crucial for accurate cardiac quantification and disease diagnosis. The related LV function indexes, such as the end-diastole volumes (EDV), the end-systole volumes (ESV), LV mass and the EF, can be computed from cardiovascular MR (CMR) image, which is the gold standard for clinical cardiac disease diagnosis.

The image segmentation technology is always used for LV function quantification in the clinical diagnosis of cardiac diseases based on the CMR. The traditional LV segmentation technology can be classified as five kinds: the method based active model [5], the method based on atlas [6], the method based on statistical model [7-9], and the method based on deep learning [10], and method based on hybrid model . Before 2011, the paper [11] has given the detailed review on the LV segmentation algorithms. After 2011, [12] proposed a method to address LV segmentation problem based on 3D active model using traditional snake model. [13] proposed a dual level set model to achieve LV segmentation. [14] proposed a method based on atlas to achieve LV segmentation as well as other tissues' segmentation on heart. However, active model and method based on atlas are the on-line optimization models. Compared with the on-line optimization model, the off-line optimization methods, such as machine learning methods, can achieve more efficient inference as well as real-time segmentation. [15] using random forest technology to achieve LV segmentation and quality estimation at the same time. Specially, deep learning method is more and more popular due to its powerful feature representation ability. Some breakthrough on LV segmentation field was achieved based on deep learning technology. For instance, [16] proposed the LV segmentation method based on fully convolutional network (FCN) for the first time. [17] proposed a regression method to achieve short-axis LV segmentation using FCN. [18] used the 3D consistent with spatial propagation to achieve LV segmentation. Additionally, some hybrid model using deep learning is also useful for LV segmentation problem. [19] combines the deep learning technology and level set into a twostage method to address the region of interest (ROI) localization and LV segmentation problem. Similarly, [20] proposed the similar hybrid technology with [19] to address LV segmentation problem.

Though a large number of methods were proposed to address LV segmentation problem on CMR image. It is still an open challenge, due to the inherent difficulties from CMR images, such as variation from motion, noisy from CMR, and subject variations. Besides, all the exiting methods ignore an important area variation correlation, 


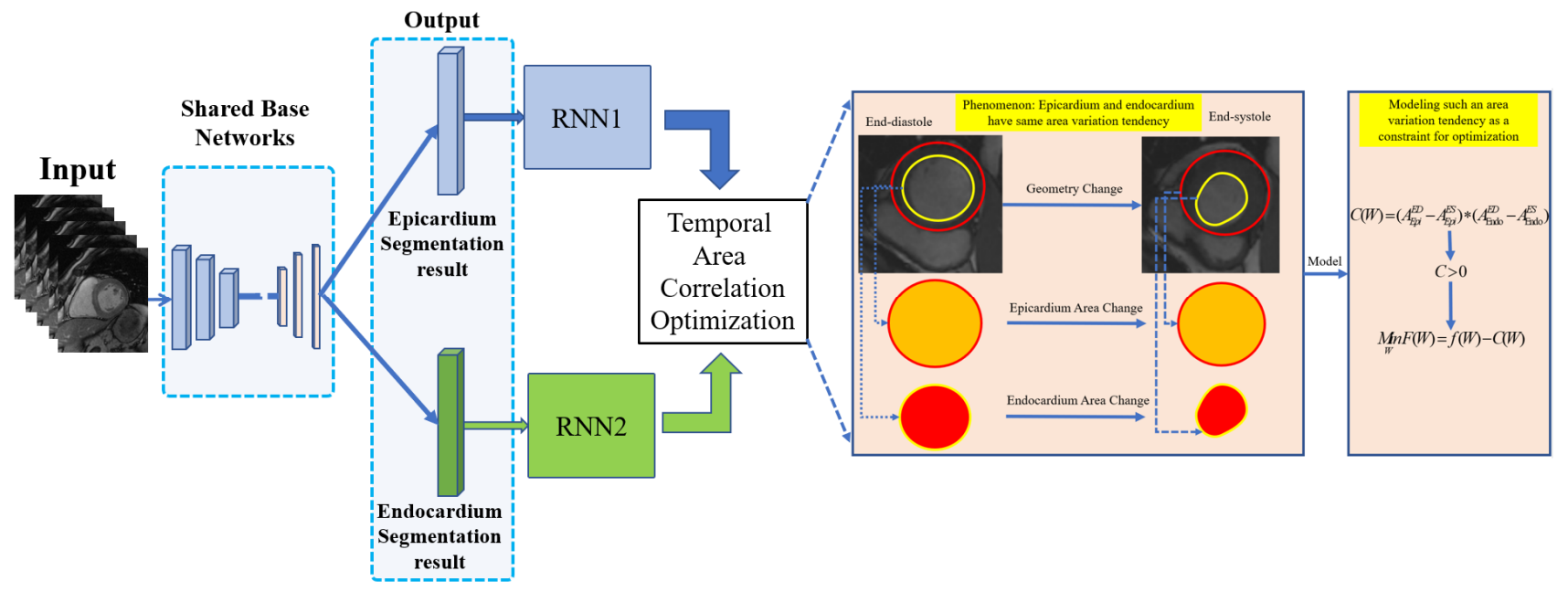

Figure 1. The framework of the proposed method

i.e., the epicardium and endocardium have the same change tendency (increasing or decreasing simultaneously).

In this paper, we proposed a new method to achieve LV segmentation based on temporal area correlation. the new attempt in large scale CMR datasets of MICCAI 2012 LV [21]. For the first time, we formulated the LV segmentation into a temporal area correlation segmentation model. Compared to the existed state-ofthe-art methods, the method achieves important improvement on performance.

\section{Methods}

\subsection{Data Preprocessing}

The CMR image preprocessing procedure includes ventricle center localization and image physical space normalization. The left ventricle center localization addressing the ROI cropping problems. The cropped CMR image around the center point can be used as the input of the network. The image physical space normalization normalizes the CMR images' physical space. This operation can provide more accurate quantification of LV function.

\subsection{Network Structure}

As shown in the Figure 1, the proposed method includes three modules: base network with encodedecode structure, the multi-task module for epicardium and endocardium segmentation, and optimization module with temporal area correlation. The base network is based on an FCN framework, which includes some convolution layers and the deconvolution layers. The base network's structure is flexible, and can be replaced by different structure, such as U-net [22], FCN in [17], or Refine-Net [23]. A large number of experiments show that the proposed framework is robust to the different of base networks. In this paper, we used the U-net as the base network to introduce the proposed temporal area correlation model based on area variation regularized deep learning network.

The multi-task module converts traditional threeclassification problem into two binary-classification problems. In this way, the area variation correlation can be modeled in the optimization module.

\subsection{Model Optimization}

The loss function can be modeled as following:

$$
L_{\text {Loss }}(W)=-\alpha \sum_{i, j}^{w, h} \ln \left(P_{\text {endo }, i, j}\right)-\beta \sum_{i, j}^{w, h} \ln \left(P_{e p i, i, j}\right)-\lambda C(W)
$$

where $W$ denotes the networks' weight, $P_{\text {endo, } i, j}$ denotes the output probability for endocardium and $P_{e p i, i, j}$ denotes the output probability for epicardium, and $C(W)$ denotes the area variation correlation for endocardium and epicardium. $P_{e n d o, i, j}$ and $P_{e p i, i, j}$ can be modeled using SoftMax activation function as following:

$$
\begin{aligned}
& P_{e p i, i, j}=e^{f_{e p i, i, j}(W)} / \sum_{c=1}^{4} e^{f_{c, i, j}(W)}, \\
& P_{\text {endo }, i, j}=e^{f_{\text {endo }, i, j}(W)} / \sum_{c=1}^{4} e^{f_{c, i, j}(W)}
\end{aligned}
$$

where $f_{e p i, i, j}(W)$ is the response value of position $(i, j)$ in the true category, $f_{e p i, i, j}(W)$ is the response value of position $(i, j)$ in the true category for epicardium, $f_{\text {endo }, i, j}(W)$ is the response value of position $(i, j)$ in the true category for endocardium. The area variation correlation $C(W)$ can be modeled as: 


$$
C(W)=\left(A_{E p i}^{E D}-A_{E p i}^{E S}\right) *\left(A_{E n d o}^{E D}-A_{E n d o}^{E S}\right)
$$

where $A_{E p i}^{E D}$ denotes the epicardium area on end-diastole, $A_{E p i}^{E S}$ denotes the epicardium area on end-systole, $A_{E n d o}^{E D}$ denotes the endocardium area on end-diastole, $A_{E n d o}^{E S}$ denotes the endocardium area on end-systole. This idea starts from the facts that the epicardium and endocardium have the same area variation tendency. They will increase or decrease simultaneously. In other words, $\left(A_{E p i}^{E D}-A_{E p i}^{E S}\right)$ and $\left(A_{E n d o}^{E D}-A_{E n d o}^{E S}\right)$ are both the positive number for negative number. Hence, $\left(A_{E p i}^{E D}-A_{E p i}^{E S}\right) *\left(A_{E n d o}^{E D}-A_{E n d o}^{E S}\right)$ is always positive number. This correlation can be further formulated and extended as the following:

$$
C(W)=\left(A_{E p i}^{T 1}-A_{E p i}^{T 2}\right) *\left(A_{E n d o}^{T 1}-A_{E n d o}^{T 2}\right)
$$

where $\mathrm{T} 1$ and $\mathrm{T} 2$ denotes the adjacent CMR image frames along the time. In this way the whole cardiac cycle's area variation correlation can be modeled. The robustness and performance can be further enhanced.

\section{Results and Discussion}

\subsection{Metrics}

The proposed method was evaluated by the widely used criterion in the LV segmentation field, including correlation coefficient (R), mean absolute errors (MAE) \pm the standard deviation (SD), Average Dice (AD) and Average Hausdorff Distance (AHD) in paper [18].

\subsection{Environment of Experiments}

We conducted the experiments in Caffe deep learning framework, which is flexible for make new temporal area variation layer. The workstation's configurations are 3.4GHz Core i7 CPU, 64GB RAM, Nvidia TiTan X (12GB memories). The codes are based on python language, and the SGD was used to train the model, the batch size is 1 for the online learning, which is the only proper way to train the model with area variation correlation. The max iteration is 30000 for training a LV segmentation model, and the Gaussian weight initializations are used to set the initial networks.

\subsection{Prediction Accuracy}

The segmentation performance and the clinical indexes quantification performance based on segmentation results have been shown in Table 1 and Table 2 respectively. As shown in the Table 1, compared with the state-of-the-art methods in LV segmentation field, the proposed method achieves the better segmentation accuracy in $\mathrm{AD}$ and AHD for both the endocardium and epicardium

Table 1. The comparison with the state-of-the-art methods on segmentation performance (PM: the

\begin{tabular}{|c|c|c|c|c|}
\hline \multirow{2}{*}{ Method } & \multicolumn{2}{|c|}{$\overline{\mathrm{AD}(\%)}$} & \multicolumn{2}{|c|}{ AHD(mm) } \\
\hline & endo & epi & endo & epi \\
\hline PM & $90 \pm 5$ & $81 \pm 5$ & $7.6 \pm 4.5$ & $9.1 \pm 1.8$ \\
\hline [16] & $85 \pm 9$ & $71 \pm 4$ & $9.7 \pm 5.2$ & $9.2 \pm 5.4$ \\
\hline [18] & $88 \pm 8$ & $81 \pm 5$ & $8.7 \pm 3.6$ & $11.9 \pm 4.5$ \\
\hline [3] & $83 \pm 5$ & $68 \pm 9$ & $11.9 \pm 5.1$ & $14.2 \pm 2.1$ \\
\hline
\end{tabular}
proposed method).

Table 2. The comparison with the state-of-the-art methods on clinical index quantification performance (PM: the proposed method).

\begin{tabular}{||c||l|l||l||l||}
\hline \multirow{2}{*}{ Method } & \multicolumn{2}{|c|}{ LV MASS } & \multicolumn{2}{c||}{ LV EF } \\
\cline { 2 - 5 } & R & MAE $(\mathrm{g})$ & R & MAE (\%) \\
\hline PM & $\mathbf{0 . 9}$ & $\mathbf{8 . 5} \pm \mathbf{4 . 1}$ & $\mathbf{0 . 9 1}$ & $\mathbf{9} \pm \mathbf{2}$ \\
{$[16]$} & 0.87 & $13.3 \pm 5.6$ & 0.87 & $9.3 \pm 6.9$ \\
{$[18]$} & 0.75 & $14.5 \pm 2.9$ & 0.87 & $11.6 \pm 3.2$ \\
{$[3]$} & 0.71 & $16.5 \pm 6.3$ & 0.85 & $12.2 \pm 3.1$ \\
\hline
\end{tabular}

segmentation. Additionally, the proposed method also achieves the best results on the clinical index quantification accuracy than the state-of-the-art method. This result also showed that the proposed method can utilize the temporal relationship between adjacent CMR frames and guarantee good consistence.

\section{Conclusion}

In the paper, we proposed a novel deep learning framework to address the LV segmentation. It introduces the temporal area variation correlation as the regularized item into end-to-end LV segmentation framework for the first time. This correlation can use the temporal area relationship between adjacent CMR frames to guarantee good consistence on segmentation results. Hence, the good performance can be achieved. The experiment results on the open accessible LV segmentation datasets prove the proposed method has the big potential to be used in the clinical diagnosis.

\section{Acknowledgements}

This work was supported by project grants from the National Natural Science Foundation of China (No. 61571165, 61572152, 61601143, and 81770328), and Science Technology and Innovation Commission of Shenzhen Municipality (No. JCYJ20151029173639477 and JSGG20160229125049615). 


\section{References}

[1] G. N. Luo, G. X. Sun, K. Q. Wang, S. Y. Dong, and H. G. Zhang, "A novel left ventricular volumes prediction method based on deep learning network in cardiac MRI," presented at the Computing in Cardiology, 2016.

[2] G. Luo, S. Dong, K. Wang, W. Zuo, S. Cao, and H. Zhang, "Multi-views Fusion CNN for Left Ventricular Volumes Estimation on Cardiac MR Images," IEEE Transactions on Biomedical Engineering, vol. 65, no. 9, pp. 1924-1934, 2018.

[3] G. N. Luo, R. An, K. Q. Wang, S. Y. Dong, and H. G. Zhang, "A deep learning network for right ventricle segmentation in short-axis MRI," presented at the Computing in Cardiology, 2016.

[4] W. Xue, A. Islam, M. Bhaduri, and S. Li, "Direct Multitype Cardiac Indices Estimation via Joint Representation and Regression Learning," IEEE Transactions on Medical Imaging, vol. 36, no. 10, pp. 2057-2067, 2017.

[5] G. N. Luo, D. Sui, K. Q. Wang, and J. Chae, "Neuron anatomy structure reconstruction based on a sliding filter," BMC Bioinformatics, vol. 16, no. 1, 2015.

[6] X. Zhuang and J. Shen, "Multi-scale patch and multimodality atlases for whole heart segmentation of MRI," Medical Image Analysis, vol. 31, pp. 77-87.

[7] H. El-Rewaidy, E.-S. Ibrahim, and A. S. Fahmy, "Segmentation of the right ventricle in MRI images using a dual active shape model," IET Image Processing, vol. 10, no. 10 , pp. $717-723$

[8] J. Wen et al., "Robust Sparse Linear Discriminant Analysis," IEEE Transactions on Circuits and Systems for Video Technology, pp. 1-1, 2018.

[9] J. Wen, N. Han, X. Fang, L. Fei, K. Yan, and S. Zhan, "Low-Rank Preserving Projection Via Graph Regularized Reconstruction," IEEE Transactions on Cybernetics, pp. 113, 2018.

[10]A. Chen et al., "Transfer Learning for the Fully Automatic Segmentation of Left Ventricle Myocardium in Porcine Cardiac Cine MR Images," in Statistical Atlases and Computational Models of the Heart. ACDC and MMWHS Challenges, Cham, 2018, pp. 21-31: Springer International Publishing.

[11]C. Petitjean and J. N. Dacher, "A review of segmentation methods in short axis cardiac MR images," (in English), Medical Image Analysis, vol. 15, no. 2, pp. 169-184, Apr 2011.

[12]M. Hajiaghayi, E. M. Groves, H. Jafarkhani, and A. Kheradvar, "A 3-D Active Contour Method for Automated Segmentation of the Left Ventricle From Magnetic Resonance Images," IEEE Transactions on Biomedical Engineering, vol. 64, no. 1, pp. 134-144, 2017.

[13]C. Yang, W. Wu, Y. Su, and S. Zhang, "Left ventricle segmentation via two-layer level sets with circular shape constraint," Magnetic Resonance Imaging, vol. 38, pp. 202213, 2017/05/01/ 2017.

[14]X. H. Zhuang and J. Shen, "Multi-scale patch and multimodality atlases for whole heart segmentation of MRI," (in English), Medical Image Analysis, vol. 31, pp. 77-87, Jul 2016.

[15]X. Albà, K. Lekadir, M. Pereañez, P. Medrano-Gracia, A. A. Young, and A. F. Frangi, "Automatic initialization and quality control of large-scale cardiac MRI segmentations,"
Medical Image Analysis, vol. 43, pp. 129-141.

[16]V. T. Phi, "A Fully Convolutional Neural Network for Cardiac Segmentation in Short-Axis MRI," arXiv, April 2016.

[17]L. K. Tan, Y. M. Liew, E. Lim, and R. A. McLaughlin, "Convolutional neural network regression for short-axis left ventricle segmentation in cardiac cine MR sequences," Medical Image Analysis, vol. 39, pp. 78-86.

[18]Q. Zheng, H. Delingette, N. Duchateau, and N. Ayache, "3D Consistent \&\#x0026;amp; Robust Segmentation of Cardiac Images by Deep Learning with Spatial Propagation," IEEE Transactions on Medical Imaging, vol. PP, no. 99, pp. 1-1, 2018.

[19]T. A. Ngo, Z. Lu, and G. Carneiro, "Combining deep learning and level set for the automated segmentation of the left ventricle of the heart from cardiac cine magnetic resonance," Medical Image Analysis, vol. 35, pp. 159-171.

[20]M. R. Avendi, A. Kheradvar, and H. Jafarkhani, "A combined deep-learning and deformable-model approach to fully automatic segmentation of the left ventricle in cardiac MRI," Med Image Anal, vol. 30, pp. 108-19, May 2016.

[21]A. Suinesiaputra et al., "A collaborative resource to build consensus for automated left ventricular segmentation of cardiac MR images,", Medical Image Analysis, vol. 18, no. 1, pp. 50-62, Jan 2014.

[22]O. Ronneberger, P. Fischer, and T. Brox, "U-Net: Convolutional Networks for Biomedical Image Segmentation,", Medical Image Computing And ComputerAssisted Intervention, Pt Iii, vol. 9351, pp. 234-241, 2015.

[23]G. Lin, A. Milan, C. Shen, and I. Reid, "RefineNet: Multipath Refinement Networks for High-Resolution Semantic Segmentation," in 2017 IEEE Conference on Computer Vision and Pattern Recognition (CVPR), 2017, pp. 51685177: IEEE.

Address for correspondence.

Kuanquan Wang

Mailbox 332, Harbin Institute of Technology

Harbin 150001, China

wangkq@hit.edu.cn 